\title{
Combined systemic and intraventricular chemotherapy in primary CNS lymphoma: a pilot study
}

U Schlegel, H Pels, A Glasmacher, R Kleinschmidt, I Schmidt-Wolf, C Helmstaedter, K Flie $\beta$ bach, $M$ Deckert, D Van Roost, R Fimmers, U Bode, T Klockgether

Abstract

The objective was to evaluate response rate, response duration, and toxicity after systemic and intraventricular chemotherapy in primary CNS lymphoma (PCNSL).

From September 1995 to September 1998, 20 consecutive patients with PCNSL (median age 64, range 27 to 71 years) were enrolled in a pilot study evaluating chemotherapy without radiotherapy. A high dose methotrexate (MTX) (cycles 1 , $2,4,5)$ and cytarabine (ara-C) (cycles 3,6 ) based systemic therapy (including dexamethasone, vinca alkaloids, ifosfamide, and cyclophosphamide) was combined with intraventricular MTX, prednisolone, and ara-C.

Complete response was achieved in 11 and partial remission in two patients; in one response could not be determined. Four patients showed progressive disease and two (70, 71 years) died from treatment related complications. Observation time was 2 to 59 months (median $\mathbf{3 1 . 5}$ months). Kaplan-Meier estimate for median time to treatment failure (TTF) was 20.5 months, and for median survival 54 months. Systemic toxicity was mainly hematological. Ommaya reservoir infection occurred in four patients and acute transient MTX induced encephalopathy in two (subacute in another). Cognitive dysfunction possibly due to treatment was seen in only one patient after relapse and after a total of 12 cycles (six at relapse).

In conclusion, primary chemotherapy based on high dose MTX and ara-C is highly efficient in PCNSL. Toxicity is manageable in patients younger than 70 years.

(F Neurol Neurosurg Psychiatry 2001;71:118-122)

Keywords: lymphoma; CNS; therapy

Biostatistics

R Fimmers

Correspondence to:

Dr U Schlegel

uwe.schlegel@uni-bonn.de

Received 11 April 2000 and in revised form

10 November 2000

Accepted 6 February 2001
Prognosis in primary CNS lymphoma (PCNSL) is poor. ${ }^{1}$ Radiotherapy alone results in a median survival of about 1 year. ${ }^{2}$ Systemic and intraventricular MTX before brain irradiation and subsequent systemic ara-C has extended median survival to about four years. ${ }^{3}$ However, careful long term evaluation of patients treated with this regimen, showed that patients over 60 years of age carry an almost $100 \%$ risk of severe neurotoxicity. ${ }^{4}$ Chemotherapy alone based on systemic MTX greater than $1 \mathrm{~g} / \mathrm{m}^{2}$ is efficient in terms of tumour response and extended survival. ${ }^{56}$ Complete response rates (median overall survival) were $65 \%$ (30.4 months) in 31 patients treated with high dose (3.5 to $8 \mathrm{~g} / \mathrm{m}^{2} /$ cycle) systemic MTX alone, ${ }^{5}$ and $65 \%$ (40.7 months) in 74 patients treated with intra-arterial cyclophosphamide and MTX (2.5 g/m²/cycle) after osmotic blood-brain barrier opening. ${ }^{7}$ In these studies, health related quality of life assessment ${ }^{5}$ and neuropsychological testing ${ }^{7}$ showed no significant long term neurotoxicity. However, more than half of the patients in the study of GuhaThakurta et al relapsed within 17 months, ${ }^{5}$ and the rationale for blood-brain barrier opening, as employed by McAllsiter et $a l^{7}$ remains controversial, because PCNSL are potentially widespread infiltrative tumours that are not always identifiable by neuroimaging. ${ }^{18}$ Here we report on the therapeutic results of 20 patients with PCNSL enrolled in a pilot study evaluating the efficacy and toxicity of a novel combined intraventricular and systemic chemotherapy regimen without radiotherapy. The study considered the questions whether systemic high dose MTX in combination with additional drugs efficient in malignant nonHodgkin's B cell lymphoma and with intraventricular chemotherapy would result in durable tumour responses with avoidance of long term neurotoxicity.

Patients and methods

Twenty consecutive HIV negative patients with PCNSL (median age 64, range 27 to 71 years) were treated with a novel chemotherapy protocol between September 1995 and September 1998, which had been derived from a paediatric oncology protocol for high grade malignant lymphoma of B cell type with initial CNS involvement. ${ }^{9}$ All immunocompetent adults, 18 to 75 years old, with a new diagnosis of PCNSL were eligible. A creatinine clearance of at least $60 \mathrm{ml} / \mathrm{min}$ was required. All patients underwent 
Table 1 Bonn chemotherapy protocol for primary CNS lymphoma

\begin{tabular}{|c|c|c|c|c|c|c|c|}
\hline Course A & Day 1 & Day 2 & Day3 & Day 4 & Day 5 & & \\
\hline Methotrexate iv $\left(5 \mathrm{~g} / \mathrm{m}^{2}\right)^{\star}$ & + & & & & & & \\
\hline Vincristine iv (2 mg) & + & & & & & & \\
\hline Ifosfamide iv $\left(800 \mathrm{mg} / \mathrm{m}^{2}\right) \dagger$ & & + & + & + & + & & \\
\hline Dexamethasone po $\left(10 \mathrm{mg} / \mathrm{m}^{2}\right)$ & & + & + & + & + & & \\
\hline Prednisolone icv $(2.5 \mathrm{mg})$ & + & + & + & + & & & \\
\hline Methotrexate icv ( $3 \mathrm{mg})$ & + & + & + & + & & & \\
\hline Ara-C icv $(30 \mathrm{mg})$ & & & & & + & & \\
\hline $\begin{array}{l}\text { Course B } \\
\text { Methotrexate iv }\left(5 \mathrm{~g} / \mathrm{m}^{2}\right)^{\star}\end{array}$ & $\begin{array}{l}\text { Day } 1 \\
+\end{array}$ & Day 2 & Day3 & Day 4 & Day 5 & & \\
\hline Vincristine iv $(2 \mathrm{mg})$ & + & & & & & & \\
\hline Cyclophosphamide iv $\left(200 \mathrm{mg} / \mathrm{m}^{2}\right) \dagger$ & & + & + & + & + & & \\
\hline Dexamethasone po $\left(10 \mathrm{mg} / \mathrm{m}^{2}\right)$ & & + & + & + & + & & \\
\hline Prednisolone icv $(2.5 \mathrm{mg})$ & + & + & + & + & & & \\
\hline Methotrexate icv (3 mg) & + & + & + & + & & & \\
\hline Ara-C icv (30 mg) & & & & & + & & \\
\hline Course $C$ & Day 1 & Day 2 & Day 3 & Day 4 & Day 5 & Day 6 & Day 7 \\
\hline Ara-C iv $\left(3 \mathrm{~g} / \mathrm{m}^{2}\right) \ddagger$ & + & + & & & & & \\
\hline Vindesine iv ( $5 \mathrm{mg})$ & + & & & & & & \\
\hline Dexamethasone po $\left(20 \mathrm{mg} / \mathrm{m}^{2}\right)$ & & & + & + & + & + & + \\
\hline Prednisolone icv $(2.5 \mathrm{mg})$ & & & + & + & + & + & \\
\hline Methotrexate icv ( $3 \mathrm{mg}$ ) & & & + & + & + & + & \\
\hline Ara-C icv (30 mg) & & & & & & & + \\
\hline
\end{tabular}

Sequence of courses: A1 (days 1-5), B1 (days 22-26), C1 (days 43-49); A2 (days 64-68), B2 (days 85-89), C2 (days 106-112). Cycles A to C (A1 to $\mathrm{C} 1$ ) are repeated once (A2 to C2).

$\star_{0.5} \mathrm{~g} / \mathrm{m}^{2}$ infusion over 30 minutes and $4.5 \mathrm{~g} / \mathrm{m}^{2}$ infusion over 23.5 hours.

$\dagger 1$ hour infusion.

$\ddagger 3$ hour infusion.

iv $=$ Intravenous; $\mathrm{po}=\mathrm{oral} ; \mathrm{icv}=$ intraventricular.

The protocol is modified in a current phase II study:

(no intraventricular therapy at day 1 of cycles A1, B1, A2, B2; systemic MTX reduced to $3 \mathrm{~g} / \mathrm{m}^{2}$ in patients $>64 \mathrm{y}$ )

bone marrow biopsy, abdominal and chest CT, lumbar puncture, and ophthalmological examination with split lamp investigation. Treatment consisted of six chemotherapy cycles separated by intervals of 2 weeks between each cycle, in which patients were off therapy. Details of the protocol are shown in table 1. Systemic MTX was administered as a 24 hour infusion under vigorous hydration and urine alkalisation; leukovorin rescue was started 10 hours after completion of MTX infusion. Ifosfamide and cyclophosphamide were given as a 1 hour infusion with uromitexan protection. High dose ara-C was given as a 3 hour infusion. Patient

Table 2 Patient data/treatment/response

\begin{tabular}{|c|c|c|c|c|c|c|c|c|}
\hline $\begin{array}{l}\text { Patient } \\
\text { No }\end{array}$ & Age & $\operatorname{Sex}$ & Modification of therapy & Response & $\begin{array}{l}\text { KPS } \\
\text { init/after }\end{array}$ & $\begin{array}{l}\text { TTF } \\
\text { (months) }\end{array}$ & Retreatment at relapse / response & $\begin{array}{l}\text { Survival } \\
\text { (months) }\end{array}$ \\
\hline 1 & 62 & M & None & CR & $70 / 90$ & 22 & $\begin{array}{l}\text { (1) CNS relapse: } 5 \text { courses systemic } \\
\text { ChT } \rightarrow \text { CR } \\
\text { (2) CNS relapse: } 4 \text { courses PCV } \rightarrow \text { PR } \\
\text { Ocular relapse: orbital RT }(20 \text { Gy }) \rightarrow \text { CR } \\
\text { (3) CNS relapse: WBRT } \rightarrow \text { PR }\end{array}$ & 54 \\
\hline 2 & 27 & $\mathrm{~W}$ & None & CR & $70 / 90$ & $59+$ & & $59+$ \\
\hline 3 & 66 & W & In A2, B2 systemic therapy with MTX $3 \mathrm{~g} / \mathrm{m}^{2}$ only & CR & $50 / 80$ & $54+$ & & $54+$ \\
\hline 4 & 39 & $\mathrm{~W}$ & None & CR & $80 / 90$ & $46+$ & & $46+$ \\
\hline 5 & 59 & W & In A2, B2 systemic therapy with MTX $3 \mathrm{~g} / \mathrm{m}^{2}$ only & CR & $70 / 90$ & $52+$ & & $52+$ \\
\hline 6 & 71 & $\mathrm{M}$ & Discontinuation after B1 because of septicaemia & PR & $60 / 0$ & 2 & & 2 \\
\hline 7 & 66 & M & None & CR & $70 / 90$ & 32 & CNS relapse: $\mathrm{PCV} \rightarrow \mathrm{PD}$; WBRT $\rightarrow \mathrm{CR}$ & $46+$ \\
\hline 8 & 65 & $\mathrm{~W}$ & None & CR & $70 / 100$ & 34 & CNS relapse: Bonn protocol $\rightarrow$ CR & $42+$ \\
\hline 9 & 51 & M & None & PR & $70 / 100$ & $33+$ & & $33+$ \\
\hline 10 & 62 & W & $\begin{array}{l}\text { No intraventricular treatment } \\
\text { In } \mathrm{A} 1,2, \mathrm{~B} 1,2 \text { systemic therapy with MTX } 3 \mathrm{~g} / \mathrm{m}^{2} \\
\text { only }\end{array}$ & CR & $60 / 80$ & 19 & $\begin{array}{l}\text { (1) CNS relapse: Bonn protocol } \rightarrow \text { CR } \\
\text { (2) CNS relapse: WBRT } \rightarrow \text { pending }\end{array}$ & $37+$ \\
\hline 11 & 69 & M & $\begin{array}{l}\text { No intraventricular treatment } \\
\text { In } \mathrm{A} 1, \mathrm{~B} 1 \text { systemic therapy with MTX } 3 \mathrm{~g} / \mathrm{m}^{2} \text { only }\end{array}$ & PD & $60 / 40$ & 0 & No further treatment & 4 \\
\hline 12 & 37 & W & $\begin{array}{l}\text { No intraventricular treatment } \\
\text { In A2, B1, } 2 \text { systemic therapy with MTX } 3 \mathrm{~g} / \mathrm{m}^{2} \\
\text { only }\end{array}$ & CR & $70 / 90$ & 6 & CNS relapse: Bonn protocol CR & $32+$ \\
\hline 13 & 59 & W & $\begin{array}{l}\text { No intraventricular treatment } \\
\text { In A2, B1, } 2 \text { systemic therapy with } \mathrm{MTX} 3 \mathrm{~g} / \mathrm{m}^{2} \\
\text { only }\end{array}$ & CR & $30 / 90$ & 9 & $\begin{array}{l}\text { Ocular relapse: Orbital RT ( } 20 \mathrm{~Gy})-\mathrm{CR} \\
\text { (1) CNS relapse: Bonn protocol } \rightarrow \mathrm{CR} \\
\text { (2) CNS relapse: } \mathrm{PCV} \rightarrow \mathrm{PD} \text {, no further } \\
\text { treatment }\end{array}$ & 31 \\
\hline 14 & 69 & M & $\begin{array}{l}\text { In A1, B1 systemic therapy with MTX } 3 \mathrm{~g} / \mathrm{m}^{2} \text { only } \\
\text { Discontinuation after C1 because of PD }\end{array}$ & $\begin{array}{l}\mathrm{PR} / \\
\mathrm{PD}\end{array}$ & $40 / 40$ & 3 & $\begin{array}{l}\text { PD: } 4 \text { courses PCV PR } \\
\text { CNS relapse: WBRT ( } 40 \text { Gy)- PR }\end{array}$ & 26 \\
\hline 15 & 65 & W & Discontinuation after B2 because of PD & $\begin{array}{l}\mathrm{PR} / \\
\mathrm{PD}\end{array}$ & $60 / 50$ & 7 & $\begin{array}{l}\text { PD: } 1 \text { course PCV } \rightarrow \text { PD } \\
\text { WBRT }(40 \text { Gy) }+ \text { focal boost }(14 \text { Gy) } \\
\text { PR }\end{array}$ & $24+$ \\
\hline 16 & 59 & M & $\begin{array}{l}\text { Discontinuation after one course (start with C1) } \\
\text { because of myelosuppression and PD }\end{array}$ & PD & $50 / 30$ & 0 & WBRT (40 Gy) $\rightarrow$ PR & 6 \\
\hline 17 & 67 & M & Systemic MTX $1.5 \mathrm{mg} / \mathrm{m}^{2}$ in cycles $\mathrm{B} 1,2$, and A2 & CR & $50 / 60$ & $28+$ & & $28+$ \\
\hline 18 & 45 & W & None & PR & $60 / 100$ & $24+$ & & $24+$ \\
\hline 19 & 67 & $\mathrm{~W}$ & None & CR & $80 / 100$ & 14 & $\begin{array}{l}\text { CNS relapse: } \mathrm{PCV} \rightarrow \mathrm{PD} \text {; WBRT (40 Gy) } \\
\mathrm{CR}\end{array}$ & $24+$ \\
\hline 20 & 70 & M & Discontinuation after A 1 because of septicaemia & PR & $50 / 0$ & 3 & & 3 \\
\hline
\end{tabular}

KPS init/after=Karnofsky performance status initially and after chemotherapy; $C R=$ complete response; $P R=$ partial response; $P D=p r o g r e s s i v e ~ d i s e a s e ; P R / P D=$ initial response with subsequent progression despite ongoing therapy; $\mathrm{ChT}=$ chemotherapy; $\mathrm{PCV}=$ combination chemotherapy with procarbazine, $\mathrm{CCNU}$ and vincristine; (WB) RT = (whole brain) radiotherapy; $\mathrm{ttf}=$ time to treatment failure. 
characteristics and modifications of therapy are given in table 2 . Tumour tissue was obtained by open biopsy/resection in nine and by stereotactic biopsy in 11 patients. All patients gave informed consent, and the study was approved by the local ethics committee. An Ommaya reservoir was placed in 19 patients; one refused to be treated intraventricularly. In three, the reservoir had to be removed before chemotherapy; in two because of reservoir infection and in another because of tumour bleeding with panventricular haemorrhage. Complete response was assumed in patients off steroids, if enhancing tumour tissue on T1 weighted MRI had disappeared (partial remission if it was reduced in volume for more than 50\%) and clinical improvement was present according to the criteria of MacDonald et al..$^{10}$ Treatment failure was defined as progressive or stable disease, relapse after response, discontinuation of chemotherapy due to complications, or death. All patients were followed up every 6 months after therapy with neurological examination, MRI, CSF examination, ophthalmological investigation, and detailed neuropsychological examination. A standardised test battery comprised standard tests of attention, verbal and non-verbal memory, verbal fluency, and visuoconstruction. To obtain a global index of cognitive function, raw data from these tests were transformed into standard values according to normative test data and then averaged. This resulted in an arbitrary score with " 100 " as a reference value for average cognitive function in a healthy person.

\section{Results}

PATIENT CHARACTERISTICS, DIAGNOSIS, AND PATHOLOGY

Brain MRI showed unilocular disease in nine and multilocular lymphoma in 11 patients. Cytology of CSF was not diagnostic. No patient presented with initial ocular involvement; however, in two, lymphoma infiltration of the uvea preceded the first (patient 13) or second (patient 1) CNS relapse. Karnofsky performance status at presentation ranged from 30 to 80 , with a median score of 70 . The PCNSL was classified as high grade malignant $\mathrm{B}$ cell lymphoma of the diffuse large cell type in 19 patients and as low grade malignant B cell lymphoma in one (patient 18). In three patients $(10,11$, and 14) systemic MTX was reduced in all cycles because of age older than 60 years and poor general condition. In five others, the MTX dose was reduced in all but the first cycle because of infection (patients 3, 5, 12, and 13) or cutaneous vasculitis (patient 17) during the first cycle. Median follow up for all patients was 31.5 months (range 2 to 59 months).

TREATMENT RESPONSE

Details of treatment response and modification of therapy due to treatment related complications are given in table 2. According to the criteria of Macdonald et al ${ }^{10} 11$ patients (55\%) achieved complete remission, and in one $(5 \%)$ a response could not be determined because of complete tumour resection. Two patients $(10 \% ; 9$ and 18$)$ with partial remission became asymptomatic under therapy but had residual

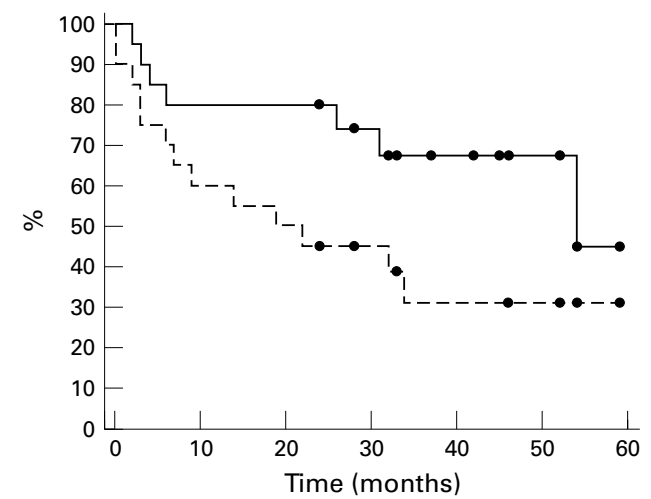

Figure 1 Kaplan-Meier analysis of survival (continuous line) and time to treatment failure (broken line) in 20 patients with PCNSL. Dots indicate last follow up.

enhancing lesions decreasing in size during follow up. All responding patients showed improvement of their Karnofsky performance status (table 2). Four patients (20\%) showed progressive disease, two of them (14 and 15) though initially showing partial remission. Two patients $(10 \%)$ died of complications related to treatment. Seven patients with complete response after therapy relapsed, and seven died (five disease and two therapy related). KaplanMeier estimates for median time to treatment failure and for median overall survival were 20.5 months and 54.0 months, respectively (fig 1).

ACUTE TOXICITY

In 99 chemotherapy cycles 41 (41\%) World Health Organisation (WHO) grade III and two $(2 \%)$ grade IV neutropenias, 17 grade III $(17 \%)$ and 10 grade IV (10\%) thrombocytopenias, and 13 grade III (13\%) and two (2\%) grade IV anaemias were encountered. Due to myelosuppression two patients died of septicaemia (6 and 20). In one patient, chemotherapy had to be discontinued (16). Two episodes of nephrotoxicity, WHO grade II; one mucositis, WHO grade III; and one vincristine induced peripheral neuropathy were transient. In patient 17 a localised cutaneous vasculitis induced by MTX made reduction of dosage necessary; one patient with a perforated duodenal ulcer after long lasting dexamethasone treatment ( 5) underwent surgery. In four patients $(2,6,12$, and, 13) infection of the Ommaya reservoir occurred. In two patients (7 and 14) a total of three acute transient episodes of encephalopathy occurred during chemotherapy with systemic MTX and ifosfamide; no acute neurotoxic episode after ara-C administration was encountered.

TREATMENT AT RELAPSE OR PROGRESSION In cases of relapse or progression salvage chemotherapy was offered: Five patients were treated at relapse again with combined systemic and intraventricular chemotherapy (Bonn protocol) as shown in table 1, one of them (1) without intraventricular therapy because of extensive confluent white matter changes, which had developed under initial therapy (see below). Reasons for administering first line therapy at relapse were a median time 
to treatment failure of more than 20 months after complete remission to initial therapy (1 and 8 ) and incomplete administration of first line therapy, most notably lack of intraventricular chemotherapy (patients 10, 12, and 13). In all five patients complete remission was achieved, followed by a second relapse in three $(12,14$, and 16 months after first relapse) and with ongoing remission in two patients ( 8 and 12). Six patients were treated by combination therapy $(\mathrm{PCV})^{11}$ with CCNU $\left(110 \mathrm{mg} / \mathrm{m}^{2)}\right.$, day 1 , procarbazine $\left(60 \mathrm{mg} / \mathrm{m}^{2} /\right.$ day $)$, days 8 to 21 , and vincristine ( $2 \mathrm{mg} /$ day), days 8,29 -at progressive disease (patients 14 and 15), at first relapse (patients 7 and 14), or second relapse (patients 1 and 13), respectively. One of them (patient 7) refused to be retreated with the Bonn protocol at first relapse despite a median time to treatment failure of 32 months. This combination therapy led to a partial remission in two patients for 5 months (patient 1), and 9 months (patient 14). In four patients, PCV resulted in progressive disease. Seven patients were irradiated. Six of them received radiotherapy after failure of salvage chemotherapy, one (patient 16) was irradiated because of progressive disease at first line therapy. In one patient response to radiotherapy is pending (patient 10), three experienced partial remission, the others complete response (table 2).

DELAYED TOXICITY

One patient (17) developed a subacute encephalopathy, which recovered over months. In five patients $(1,8,9,13$, and 16) T2 weighted MRI showed confluent white matter lesions suggestive of chemotherapy induced leukoencephalopathy, developing 2 (patients 1 and 16) and 6 months (patients 8, 9, and 20) after initiation of therapy. One patient (13) developed similar changes 1 month after initiation of combined intraventricular/systemic chemotherapy for first relapse. Four of the patients showed no cognitive impairment, patient 16 died of disease, and patient 13 had severe cognitive dysfunction 21 months after initial diagnosis. This patient had received 12 chemotherapy cycles (six for relapse) and irradiation of the posterior chamber of the eye (20 Gy) for bilateral lymphomatous chorioretinitis. She died of a second CNS relapse 31 months after diagnosis. All other patients showed either preserved-if normal at presentation-or improved cognitive function after therapy and during follow up. In 10 patients (1-5, 7-9, $17,18)$ neuropsychological test results were documented at therapy, 4 months, 12 months, and at a third time point (15 to 41 months) after completion of treatment: All of them showed a stable or increasing summary score of cognitive function with a median of 95 (range 89 to 107) at last follow up (with 100 as a reference value for average cognitive function).

\section{Discussion}

The patients enrolled in this pilot study represent an unselected group of patients with PCNSL comparable with other patient populations in treatment studies of PCNSL. Our results suggest that long lasting responses can be achieved with chemotherapy alone in a substantial fraction of patients. We found a median time to treatment failure of 20.5 months, a median overall survival of 54 months, and a 2 year survival fraction of $80 \%$. These results are superior to those achievable with radiotherapy alone $^{2}$ and as good as or superior to results reported in three other unicentre "chemotherapy only" trials in PCNSL. Employing systemic high dose MTX alone Guha-Thakurta et al reported a $100 \%$ response rate; however, median progression free survival was 16.7 and median overall survival only 30.4 months in 31 patients. ${ }^{5}$ In 74 patients treated with intraarterial cyclophophamide and high dose MTX after blood-brain barrier opening a median overall survival of 40.7 months has been reported without long term neurotoxicity as documented by serial neurosychological testing in 23 out of these 74 patients with ongoing complete remission. ${ }^{7}$ However, this treatment modality is invasive, requires general anaesthesia, and is associated with potential acute neurotoxicity (seizures in $6 \%$ to $8 \%$ of the procedures, strokes in $6.8 \%$ of the patients). ${ }^{7}$ In a combined pilot/phase II study on 14 patients treated with systemic high dose MTX, thiotepa, vincristine, dexamethasone, and intraventricular MTX and ara-C a 79\% complete remission has been achieved with a median progression free survival of 16.5 months and a projected minimum median overall survival of 40 months. ${ }^{6}$ Six out of 14 patients had received radiotherapy before chemotherapy or at relapse; two out of eight treated with chemotherapy alone developed leukoencephalopathy, most likely attributable to treatment. ${ }^{6}$

Intraventricular chemotherapy was administered in the present study as PCNSL has a notorious affinity to the leptomeninges ${ }^{3}$ and because repeated low dosages of MTX via an Ommaya reservoir favour an even and sustained drug concentration in the CSF..$^{12}$ In fact, three patients with complete response to systemic chemotherapy alone had an early relapse and achieved a complete response again after being retreated with combination intraventricular and systemic chemotherapy. However, it cannot be proved by these data, that intraventricular therapy is of benefit, because initial systemic chemotherapy dosage had been reduced in these three patients as well. Ommaya reservoir implantation was associated with infection in four of 19 patients, which is a higher frequency than reported in other series. $^{3613}$ The reasons for this finding may be twofold: (1) Many patients of this series had been referred to our centre in a severely immunocompromised condition, most often due to prolonged treatment with steroids. (2) The present protocol is aggressive resulting in myelosuppression within a few days. If in a phase II trial with the Bonn protocol Ommaya reservoir infections cannot be avoided, intraventricular therapy would no longer be justified. Despite a pharmakokinetic rationale for this modality, a clinical proof for its efficacy in PCNSL is still lacking; beneficial treatment results may even be hampered by therapy induced ventriculitis. 
The use of vinca alkaloids and alkylating agents in PCNSL may also be questioned because these substances do not readily cross the blood-brain barrier. However, a large tumour volume in PCNSL is characterised by blood-brain barrier breakdown, and vinca alkaloids as well as alkylating agents are efficient drugs in high grade lymphomas. General toxicity is considerable with the Bonn protocol. Treatment related myelosuppression, WHO grade IV infection with treatment discontinuation, and treatment related deaths occurred soon after the first treatment cycle or under ara-C based therapy. Therefore, future trials will have to focus on intensified supportive measures - for example, GCSF prophylaxis and possibly on dose de-escalation to minimise treatment related complications.

In the present series fully reversible transient, probably MTX induced, encephalopathies were encountered in three patients and white matter lesions in six similar to those reported in patients treated with high dose systemic MTX for sarcomas. These lesions are usually asymptomatic, ${ }^{14}$ but it remains to be determined whether cognitive function will remain normal in our patients with white matter lesions. Intraventricular MTX was given together with systemic MTX at day 1 of MTX based chemotherapy cycles. This is probably dispensable, because high dose systemic MTX will result in cytotoxic CSF concentrations for 24 to 72 hours. ${ }^{15}$ To further reduce the likelihood of neurotoxic side effects, intraventricular MTX administration at day 1 has been abandoned in all patients and systemic MTX reduced to $3 \mathrm{~g} / \mathrm{m}^{2}$ in patients older than 64 in an ongoing phase II study. Long term treatment related neurotoxicity is a fundamental issue in PCNSL. ${ }^{3}{ }^{416}$ Severe cognitive dysfunction, leukoencephalopathy, or brain atrophy developed in all patients over 60, 6 months to 4 years after systemic and intraventricular MTX, radiotherapy, and postradiotherapy ara-C, which may be a consequence of this particularly neurotoxic treatment combination. ${ }^{4} \mathrm{How}-$ ever, recent reports on systemic MTX alone before radiotherapy reported a considerable rate of late neurotoxic effects as well. Five out of 15 patients older than $60(33 \%)$ showed progressive diffuse leukoencephalopathy by MRI after short infusion high dose MTX and consecutive whole brain radiotherapy ${ }^{17}$ and six out of $39(15 \%)$ patients treated with 2 cycles of MTX $\left(1 \mathrm{~g} / \mathrm{m}^{2}\right)$ before whole brain radiotherapy in a recent phase II study showed a "dementing illness". ${ }^{18} \mathrm{~A}$ high risk of neurotoxicity in older patients due to combination radiotherapy and chemotherapy was impressively underscored by the recent results of Abrey et al, who reported a rate of $10 / 12$ patients $(83 \%)$ with severe late neurotoxicity after MTX based chemotherapy followed by radiotherapy, whereas none out of 20 patients older than 60 developed neurotoxicity after chemotherapy alone. ${ }^{19}$ Because both treatment groups showed an identical median overall survival, these results suggest that systematic evaluation of chemotherapy only protocols in phase II trials would be useful.

1 Maher EA, Fine HA. Primary CNS lymphoma. Semin Oncol 1999;26:346-56.

2 Nelson DF, Martz KL, Bonner H, et al. Non-Hodgkin's lymphoma of the brain: can high dose, large volume therapy improve survival? Report on a prospective trial by the Radiation Therapy Oncology Group (RTOG): RTOG 8315. Int $\mathcal{F}$ Radiat Oncol Biol Phys 1992;23:9-17.

3 DeAngelis LM, Yahalom J, Thaler HT, et al. Combined modality therapy for primary CNS lymphoma. 7 Clin Oncol 1992;10:635-43.

4 Abrey LE, DeAngelis LM, Yahalom J. Long-term survival in primary CNS lymphoma. F Clin Oncol 1998;16:859-63.

5 Guha-Thakurta N, Damek D, Pollack C, et al. Intravenous methotrexate as initial treatment for primary central nervmethotrexate as initial treatment for primary central nervous system lymphoma: response to therapy and
life of patients. $\mathcal{F}$ Neurooncol 1999;43:259-68.

life of patients. $\mathcal{F}$ Neurooncol 1999; 43:259-68.
6 Sandor V, Stark-Vancs V, Pearson D, et al. Phase II trial of chemotherapy alone for primary CNS and intraocular lymphoma. F Clin Oncol 1998;16:3000-6.

7 McAllister LD, Doolittle ND, Guastadisegni PE, et al. Cognitive outcomes and long-term follow-up results after enhanced chemotherapy delivery for primary central nervous system lymphoma. Neurosurgery 2000;46:51-61.

8 DeAngelis LM. Primary central nervous system lymphoma: curable without toxicity? Cancer fournal from Scientific American 1996;2:137-9.

9 Reiter A, Schrappe M, Tiemann M, et al. Improved treatment results in childhood B-cell neoplasms with tailored intensification of therapy: a report of the Berlin-Frankfurt-Münster group trial NHL-BFM 90 . Blood 1999;10:3294-306.

10 Macdonald DR, Cascino TL, Schold SC, et al. Response criteria for phase II studies of supra-tentorial glioma. 7 Clin Oncol 1990;8:1277-80.

11 Cairncross JG, Macdonald DR, Ludwin S et al. Chemotherapy for anaplastic oligodendroglioma. 7 Clin Oncol 1994;12:2013-21.

12 Bleyer WA, Poplack DG, Simon RM. Concentration $\times$ time methotrexate via a subcutaneous reservoir: a less toxic regimen for intraventricular chemotherapy of central nervous system neoplasms. Blood 1978;51:835-42.

13 Sandberg DI, Bilsky MH, Souweidane MM, et al. Ommaya reservoirs for the treatment of leptomeningeal metastases. Neurosurgery 2000;47:49-55.

14 Lien HH, Blomlie V, Saeter G, et al. Osteogenic sarcoma: MR signal abnormalities of the brain in asymptomatic patients treated with high-dose methotrexate. Radiology 1991;179:547-50

15 Glantz MJ, Cole BF, Recht L, et al. High-dose intravenous methotrexate for patients with non-leukemic leptomeningeal cancer: is intrathecal chemotherapy necessary? $\mathcal{f}$ Clin Oncol 1998;16:1561-7.

16 Schlegel U, Pels H, Oehring R, et al. Neurologic sequelae of treatment of primary CNS lymphomas. F Neurooncol 1999; 43:277-86.

17 Hiraga S, Arita N, Ohnishi T et al. Rapid infusion of high-dose methotrexate resulting in enhanced penetration into cerebrospinal fluid and intensified tumor response in primary central nervous system lymphomas. 7 Neurosurg 1999;91:221-30.

18 O'Brien P, Roos D, Pratt G et al. Phase II multicenter study of brief single-agent methotrexate followed by irradiation in primary CN lymhoma. 7 Clin Oncol 2000;18:519-26.

19 Abrey LE, Yahalom J, DeAngelis LM. Treatment for primary CNS lymphoma: The next step. $\mathcal{f}$ Clin Oncol 2000; 18:3144-50. 